\title{
Die Polarisation der quasi-monodhromatischen Bremsstrahlung von einem Einkristall*
}

\author{
Von H. Überall \\ Harrison M. Randall Laboratory of Physics, University of Michigan, Ann Arbor, Michigan, USA \\ (Z. Naturforschg. 17 a, 332-334 [1962] ; eingegangen am 8. März 1962)
}

\begin{abstract}
Es wird gezeigt, daß die kürzlich von Diambrini und Mitarbeitern aufgefundene quasi-monochromatische Bremsstrahlung, welche von energetischen Elektronen bei der Durchquerung eines Einkristalls abgegeben wird, in hohem Grad linear polarisiert ist.
\end{abstract}

Bereits 1935 wurden von Williams Kohärenzeffekte der Bremsstrahlung in einem Kristall vorhergesagt ${ }^{1}$. In später erschienenen Arbeiten ${ }^{2,3}$, darunter auch vom Verfasser ${ }^{4}$, wurde der Versuch unternommen, eine quantitative Theorie dieses Phänomens aufzustellen; eine einfache, anschauliche Darstellung wurde ferner von $\mathrm{FRISCH}_{\text {gegeben }}{ }^{5 a}$ und eine Überprüfung der Grundlagen wurde von $\mathrm{S}_{\mathrm{CHIFF}}{ }^{5 \mathrm{~b}}$ angestellt. In experimentellen Studien des Effekts, welcher erst bei primären Elektronenenergien von mehr als $200 \mathrm{MeV}$ wesentlich in Erscheinung tritt, konnte derselbe neuerdings mittels moderner Elektronenbeschleuniger eindeutig nachgewiesen werden ${ }^{6-8}$.

Wie gezeigt wurde ${ }^{4}$, besteht die Wirkung des Kristalls darin, den differentiellen Bremsstrahlungsquerschnitt ${ }^{9}$ mit dem Ausdruck

$\left.\left.\exp \left(-A q^{2}\right)\right|_{\bar{\Sigma}} \exp (i q \cdot \Omega)\right|^{2}+N\left[1-\exp \left(-A q^{2}\right)\right]$

zu multiplizieren, wobei q den während des Prozesses übertragenen Impuls darstellt, sowie $\exp \left(-A q^{2}\right)$ den Debye-Wallerschen Temperaturfaktor, und wo die Summe über alle Gitterpunkte $\mathfrak{Q}$, deren Anzahl $N$ betrage, zu erstrecken ist. Das Absolutquadrat der Summe liefert die Interferenzen, und bewirkt, daß der Vektor q nur die Werte $2 \pi \mathfrak{g}$ annehmen kann, wobei g alle reziproken Gitterpunkte durchläuft. Die übliche Integration über den $\mathfrak{q}$-Raum, die zur Erlangung des totalen Querschnitts gemacht werden

\footnotetext{
* Supported in part by the Office of Naval Research, United States Navy.

1 E. J. Williams, K. Danske Vidensk. Selsk. Mat-fys. Medd. 13, 4 [1935].

2 B. Ferretti, Nuovo Cim. 7, 118 [1950].

3 M. L. Ter-Mikaelyan, J. Exp. Theor. Phys. 25, 296 [1953].

${ }^{4}$ H. Úberall, Phys. Rev. 103, 1055 [1956].

5a O. R. Frisch, Acta Phys. Austriaca 12, 331 [1959].

5b L. I. Schiff, Phys. Rev. 117, 1394 [1960].
}

muß, geht also in eine Summe über das reziproke Gitter über. Im q-Raum kommt der überwiegende Beitrag zum differentiellen Wirkungsquerschnitt von einem flachen Bereich ${ }^{4}$, welcher sich senkrecht zur Einfallsrichtung der Elektronen über eine Distanz $m c$ erstreckt ( $m=$ Elektronenmasse), jedoch eine Dicke von nur $\delta m c$ besitzt, wobei $\delta \equiv k /\left(2 \varepsilon_{1} \varepsilon_{2}\right)$ den Mindestwert des übertragenen Impulses bedeutet, wobei $\varepsilon_{1}, \varepsilon_{2}$ und $k$ die in Einheiten von $m c^{2}$ gemessenen Energien des Primär- und Sekundärelektrons und des Photons sind. Im Kristall tragen dann nur die innerhalb dieses Bereichs liegenden reziproken Gitterpunkte zum Wirkungsquerschnitt bei; das sind dann hauptsächlich die Punkte der durch den Ursprung gehenden, zur Einfallsrichtung ungefähr normalen reziproken Gitterebene, weil zum Auftreten der Kohärenzeffekte sehr kleine Einfallswinkel bezüglich einer Gitterachse gewählt werden müssen. In der Rechnung ${ }^{4}$ wurde daher der Einfachheit halber diese Ebene reziproker Gitterpunkte durch eine kontinuierliche Ebene ersetzt; diese Annahme erscheint für nicht zu hohe Primärenergien $\varepsilon_{1}$ und nicht zu geringe relative Bremsstrahlungsenergien $x \equiv k / \varepsilon_{1}$ gerechtfertigt.

Geht man jedoch zu höheren Energien, $\varepsilon_{1} \gtrsim 1 \mathrm{GeV}$, was mit den heutigen Elektronenbeschleunigern möglich ist, so wird obige vereinfachende Annahme nicht mehr zulässig. Diese Tatsache wurde vor kurzem von Diambrini und Mitarbeitern ${ }^{10}$ im Zusammen-

6 O.R.Frisch u. D.N.Olson, Phys. Rev. Letters 3, 141 [1959].

7 A. N. Saxena, Phys. Rev. Letters 4, 311 [1960]; Phys. Rev. 125, 1720 [1962]

8 G. Bologna, G. Diambrini u. G. P. Murtas, Phys. Rev. Letters 4, 572 [1960].

9 W. Heitler, The Quantum Theory of Radiation, Oxford University Press, New York 1954.

10 G. Barbiellini, G. Bologna, G. Diambrini u. G. P. Murtas, Phys. Rev. Letters 8, 112 [1962]. 
hang mit ihren sehr genauen Messungen entdeckt; anschließend angestellte Rechnungen dieser Physiker ergaben das höchst interessante Resultat, daß an Stelle eines mit abnehmendem $x$ monoton anwachsenden Interferenzbeitrages zum Bremsstrahlungsspektrum ${ }^{4}$ eine hohe und ziemlich scharfe "Linie“" im weichen Teil des Spektrums auftritt, die den Beitrag des reziproken Gitterpunktes darstellt, welcher dem Ursprung des reziproken Gitters am nächsten liegt; dazu kommen noch bei größerem $x$ liegende weitere diskontinuierliche Maxima geringerer Höhe, von entfernter liegenden reziproken Gitterpunkten verursacht. Infolge dieser Entdeckung Diambrinis erscheint es möglich, hochenergetische quasi-monochromatische Bremsstrahlung zu erzeugen.

Da die kohärente Bremsstrahlung linear polarisiert ist ${ }^{11}$, so wird dies auch für die quasi-monochromatische Linie der Fall sein. Im folgenden werden die Ergebnisse einer Berechnung der linearen Polarisation dieser Linie angegeben, welche nach dem Vorgang der früheren Rechnungen des Verfassers $^{4,11}$ ausgeführt wurde. Danach erhielten wir für das Bremsstrahlungsspektrum die Formel

$$
\begin{gathered}
\frac{\mathrm{d} \sigma}{\mathrm{d} k}=\frac{Z^{2}}{137}\left(\frac{e^{2}}{m c^{2}}\right)^{2} \frac{N}{k} \Phi(x), \\
\Phi(x)=\left[1+(1-x)^{2}\right]\left(\Psi_{1}{ }^{\mathrm{C}}+\sum_{i} \Psi_{1}^{i}\right) \\
-\frac{2}{3}(1-x)\left(\Psi_{2}{ }^{\mathrm{C}}+\frac{\sum_{i}}{\left.\Psi_{2}{ }^{i}\right)} ;\right. \\
\Psi_{1}^{\mathrm{C}}=2(2 \ln \beta+S+2), \\
\Psi_{2}{ }^{\mathrm{C}}=2(2 \ln \beta+S+3), \\
S=(1+D) e^{D} \operatorname{Ei}(-D), \\
D=\beta^{-2} A, \quad \beta=111 Z^{-1 / 3},
\end{gathered}
$$

mit der Kernladungszahl $Z$, dem elektrischen Elementarquantum $e$, dem Exponentialintegral Ei und der Konstanten $A$ des Debye-Waller-Faktors, definiert in der oben zitierten Arbeit ${ }^{4}$. Die Funktionen $\Psi_{k}{ }^{\mathrm{C}}$ sind der Beitrag des zweiten Gliedes in Formel (1) ; das erste Glied liefert einen Beitrag, welcher für den Fall eines Diamantkristalles (mit trivialen Änderungen für andere Kristallstrukturen) folgendermaßen lautet:

$$
\begin{aligned}
& \Psi_{1}{ }^{i}=\frac{1}{8} \mathscr{S}^{2}\left(\frac{2 \pi}{a}\right)^{2} \frac{4 \delta}{a} \frac{\exp \left(-A b_{i}{ }^{2}\right)}{\left.\left(\beta^{-2}+b_{i}\right)^{2}\right)^{2}} \frac{b_{i}{ }^{2}}{q_{z i}{ }^{2}}, \quad \text { (6 a) } \\
& \Psi_{2}{ }^{i}=\frac{1}{8} \mathscr{S}^{2}\left(\frac{2 \pi}{a}\right)^{2} \frac{4 \delta}{a} \frac{\exp \left(-A b_{i}{ }^{2}\right)}{\left.\left(\beta^{-2}+b_{i}\right)^{2}\right)^{2}} \frac{6 b_{i}{ }^{2}}{q_{z i^{4}}} \delta\left(q_{z i}-\delta\right) ;
\end{aligned}
$$

${ }^{11}$ H. Überalt, Phys. Rev. 107, 223 [1957]. $a$ ist die Kantenlänge des fundamentalen Gitterwürfels, gemessen in Elektronen-Сомртол-Wellenlängen; $b_{i}$ ist der $2 \pi$-fache Abstand der reziproken Gitterpunkte vom Ursprung, und das kleinste $b_{i}$ liefert den größten Beitrag zum Interferenzeffekt. Ferner ist

$$
q_{z i}=-b_{i} \vartheta \cos \varphi_{i}
$$

wobei der einfallende Elektronenstrahl $p_{1}$ den Winkel $\vartheta$ mit einer Gitterachse a bildet und $\varphi_{i}$ das Azimut zwischen der $\mathfrak{p}_{1}, \mathfrak{a}$-Ebene und der $\mathfrak{p}_{1}, \mathfrak{b}_{i}$-Ebene bedeutet, wobei $\mathfrak{b}_{i}=2 \pi \mathrm{g}$. Die relative Bremsstrahlungsenergie muß die Ungleichung erfüllen:

$$
x \leqq \frac{-2 \varepsilon_{1} b_{i}}{1-2 \varepsilon_{1} b_{i}} \frac{\vartheta \cos \varphi_{i}-b_{\mathrm{i}}{ }^{2}}{\vartheta \cos \varphi_{i}-b_{i}{ }^{2}},
$$

andernfalls sind die entsprechenden Funktionen $\Psi_{k}^{i}$ gleich Null zu setzen. Das Gleichheitszeichen in (8) liefert die oberen Kanten der "Interferenzlinien“" im Bremsstrahlungsspektrum.

Der numerische Faktor $|\mathscr{S}|^{2} / 8$ in (6) kommt im Falle des Diamantkristalles so zustande: das Gitter besteht aus zwei kubisch-flächenzentrierten Teilgittern, welche um den Vektor $a\left(\frac{1}{4}, \frac{1}{4}, \frac{1}{4}\right)$ gegeneinander versetzt sind; der Fundamentalwürfel enthält somit 8 Atome. Infolgedessen wird der LAUE-BraGGFaktor

$$
\left|\sum_{\mathfrak{L}} \exp (i q \cdot \mathfrak{Q})\right|^{2}
$$

in Gl. (1) ein Produkt aus dem üblichen kubischen LaUe-Bragg-Faktor und einem Strukturfoktor $\mathscr{S}$,

$$
\left|\sum_{\mathbb{I}} \exp (i \mathfrak{q} \cdot \mathfrak{Q})\right|^{2}=N^{\prime}\left(\frac{2 \pi}{a}\right)^{3} \sum_{\mathfrak{g}^{\prime}} \delta\left(\mathfrak{q}-2 \pi \mathfrak{g}^{\prime}\right) \mid \mathscr{S}^{2}
$$

mit $N^{\prime}$ der Anzahl der Fundamentalwürfel im Kristall, $N^{\prime}=N / 8$, und den kubischen reziproken Gittervektoren

$$
\mathfrak{g}^{\prime}=\frac{1}{a}\left(n_{1}, n_{2}, n_{3}\right)
$$

(die Summe läuft über alle ganzzahligen Wertetripel $n_{1} n_{2} n_{3}$ ), von denen jedoch viele durch den Strukturfaktor

$$
\begin{aligned}
& \mathscr{S}=\left(1+\exp \left\{i \pi\left(n_{1}+n_{2}\right)\right\}+\exp \left\{i \pi\left(n_{2}+n_{3}\right)\right\}\right. \\
& \left.+\exp \left\{i \pi\left(n_{3}+n_{1}\right)\right\}\right) \cdot\left(1+\exp \left\{i \pi\left(n_{1}+n_{2}+n_{3}\right) / 2\right\}\right)
\end{aligned}
$$

ausgelöscht werden. Für den dem Ursprung nächstliegenden, nicht gelöschten reziproken Gitterpunkt

$$
\mathfrak{b}_{1}=\frac{2 \pi}{a}(0,0,4)
$$


[Einfallsrichtung nahe einer dichtbesetzten Atomreihe $\mathfrak{a}$ in der (001)-Ebene; man wähle am besten auch $\varphi_{1}=\pi$ ] beträgt der Strukturfaktor $\mid \mathscr{S}^{2}=64$.

Die Linearpolarisation der Bremsstrahlung ist definiert als

$$
P=\left(\sigma_{\perp}-\sigma_{\|}\right) /\left(\sigma_{\perp}+\sigma_{\|}\right)
$$

mit $\sigma_{\perp}+\sigma_{\|}=\sigma$, wobei $\sigma_{\perp}(\sigma \|)$ den differentiellen oder totalen Wirkungsquerschnitt mit dem elektrischen Vektor der Bremsstrahlung senkrecht (parallel) zur $\mathfrak{a}, p_{1}$-Ebene bedeutet. Wir erhalten für sie den Ausdruck

$$
P=2(1-x) \sum_{i} \Psi_{3}^{i} / \Phi(x)
$$

mit

$$
\Psi_{3}^{i}=-\left(\delta^{2} / q_{z i}{ }^{2}\right) \Psi_{1}^{i} \cos 2 \varphi_{i} .
$$

Numerische Resultate für die quasi-monochromatische Linie des Spektrums (Beitrag des reziproken Gitterpunktes $\mathfrak{b}_{1}$ ) und ihre Polarisation wurden unter den folgenden Annahmen gewonnen: in einen

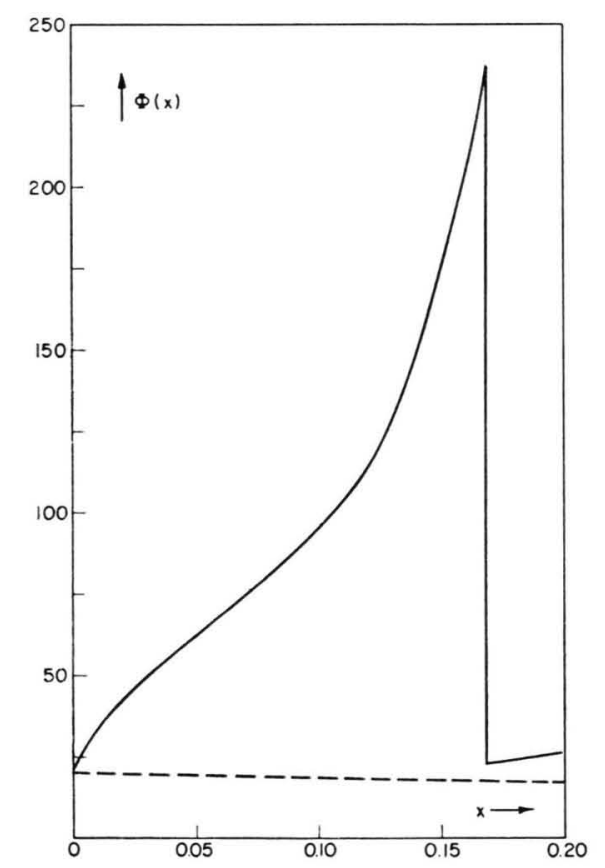

Abb. 1. Bremsstrahlungsspektrum, aufgetragen gegen die relative Photonenenergie $x=k / \varepsilon_{1}$, für eine Primärelektronenenergie von $\varepsilon_{1}=1,17 \cdot 10^{4}(6 \mathrm{GeV})$, wobei die Primärelektronen unter einem Winkel $\vartheta=0,31$ Milliradian gegen eine Achse in der (001)-Ebene eines auf Zimmertemperatur befindlichen Diamantkristalles einfallen, und zwar so, daß die Einfallsebene die [001]-Achse enthält. Die gebrochene Linie gibt den Beitrag des zweiten Gliedes in Gl.(1) zum Spektrum.
Diamantkristall bei Zimmertemperatur (entsprechender Wert von $A$ aus Tafel II der zitierten Arbeit ${ }^{4}$ ) falle ein Elektronenstrahl von $6 \mathrm{GeV}$ Energie unter einem Winkel von $\vartheta=0,31$ Milliradian gegen $z$. B. die [110]-Achse ein, mit einem Azimut $\varphi_{1}=\pi[\mathrm{d}$. h. in der (110)-Ebene]. Die Bedingung (8) schneidet dann das mit wachsendem $x$ ansteigende Spektrum bei $k=1 \mathrm{GeV}$ diskontinuierlich ab. Die entsprechende „Linie“ ist in Abb. 1 dargestellt, und Abb. 2 zeigt ihre Polarisation, welche bis zu $|P|=90 \%$ reicht.

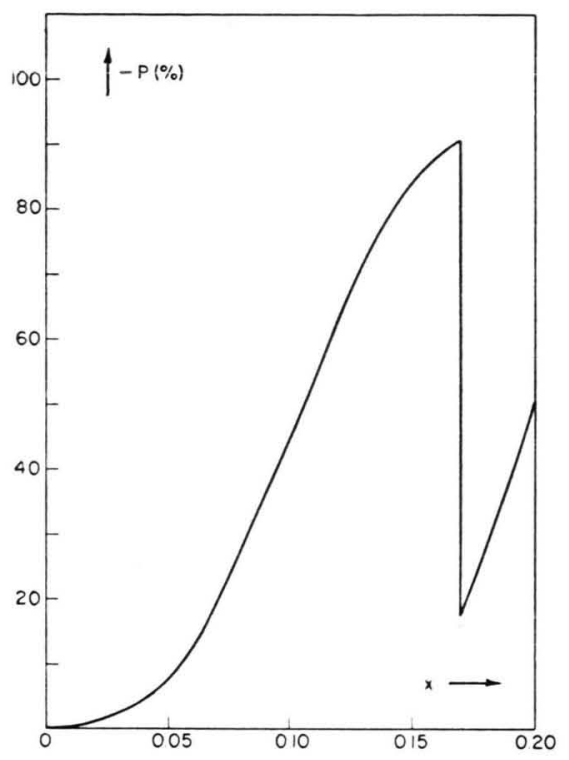

Abb. 2. Lineare Polarisation des Bremsstrahlungsspektrums von Abb. 1, aufgetragen gegen die relative Photonenenergie.

Die weiter entfernten reziproken Gitterpunkte $\mathfrak{b}_{i}$ verursachen ähnliche unstetige Zacken wie in Abb. 1 , bei höheren Photonenenergien $k$ gelegen und von viel geringerer Größe (vgl. die oben zitierte Arbeit ${ }^{10}$ ).

Abschließend möchten wir wiederholen, daß diese von Diambrini und Mitarbeitern gefundenen Unstetigkeitseffekte im Bremsstrahlungsspektrum von einem Einkristall eine große Bedeutung für die Erzeugung quasi-monochromatischer hochenergetischer $\gamma$-Strahlung besitzen. Wie wir in der vorliegenden Arbeit zeigten, ist diese quasi-monochromatische Strahlung auch sehr stark linear polarisiert.

Herrn John J. Bowman bin ich für die Durchsicht der Rechnungen zu Dank verpflichtet. 